ARTIGOS

Submetido 30.07.2020. Aprovado 09.03.2021

Avaliado pelo sistema double blind review. Editor Científico: Henrique Correa

Versão traduzida | DOI: http://dx.doi.org/10.1590/S0034-759020220106x

\title{
SUA CADEIA DE SUPRIMENTOS ESTÁ PREPARADA PARA A PRÓXIMA INTERRUPÇÃO? CONSTRUINDO CADEIAS RESILIENTES
}

\author{
Is your supply chain ready for the next disruption? Building resilient chains \\ ¿Su cadena de suministro está preparada para la próxima disrupción? Construcción de cadenas \\ resilientes
}

\author{
Murilo Zamboni Alvarenga1 ${ }^{1}$ murilozamboni@hotmail.com | ORCID: 0000-0002-4349-8262 \\ Marcos Paulo Valadares de Oliveira ${ }^{1}$ | marcos.p.oliveira@ufes.br | ORCID: 0000-0003-2646-5247 \\ Hélio Zanquetto Filho ${ }^{1}$ | zanquetto@gmail.com | ORCID: 0000-0002-5693-0704 \\ Kevin C. Desouza² | kevin.desouza@qut.edu.au | ORCID: 0000-0002-4734-3081 \\ Paula Santos Ceryno3 | paula.ceryno@unirio.br | ORCID: 00oo-0o01-8185-9092 \\ ${ }^{1}$ Universidade Federal do Espírito Santo, Programa de Pós-Graduação em Administração, Vitória, Espírito Santo, Brasil \\ ${ }^{2}$ Queensland University of Technology, School of Management, Brisbane, Queensland, Austrália \\ 3Universidade Federal do Estado do Rio de Janeiro, Departamento de Engenharia de Produção, Rio de Janeiro, Rio de Janeiro, Brasil
}

\section{RESUMO}

A habilidade em se recuperar de interrupções é um importante fator para as organizações e suas cadeias de suprimentos. Dados empíricos foram utilizados para investigar fatores que impactam a recuperação das cadeias de suprimentos em face de interrupções, como a colaboração, visibilidade, flexibilidade, orientação analítica e a gestão de riscos. Uma revisão de literatura foi conduzida para desenvolver o questionário que foi aplicado em indústrias de transformação do Brasil. Os métodos estatísticos utilizados incluem a análise fatorial confirmatória e a modelagem de equações estruturais. Os resultados indicam que o pacote de capabilidades em resiliência - colaboração, flexibilidade, visibilidade e orientação analítica - impacta positivamente a resiliência das cadeias de suprimentos. Assim, aprimorar essas capabilidades permitirá uma recuperação mais adequada de interrupções. Também se constatou que a gestão de riscos, sozinha, não favorece a recuperação de interrupções. Adicionalmente, existe um impacto mútuo entre as capabilidades em resiliência e a gestão de riscos.

PALAVRAS-CHAVE | Resiliência em cadeias de suprimentos, capabilidades de resiliência, gestão de riscos, recuperação, modelagem de equações estruturais.

\section{ABSTRACT}

The ability to recover from disruptions is important for organizations and supply chains. Empirical data were used to investigate factors that affect supply chain recovery from disruptions, including collaboration, visibility, flexibility, analytical orientation, and supply chain risk management. A literature review was conducted to build an online questionnaire that was applied to manufacturing firms in Brazil. This work's statistical method includes confirmatory factor analysis and structural equation modeling. Our results indicate that a package of resilience capabilities - collaboration, flexibility, visibility, and analytical orientation - positively affect supply chain resilience. Improving such capabilities, therefore, will allow supply chains to recover better from disruptions. It was also discovered, however, that supply chains do not recover from disruptions by way of supply chain risk management alone. Mutual impacts also exist between the group of resilience capabilities and supply chain risk management.

KEYWORDS I Supply chain resilience, resilience capabilities, supply chain risk management, recovery, structural equation modeling.

\section{RESUMEN}

La capacidad de recuperarse de las disrupciones es un factor importante para las organizaciones y sus cadenas de suministro. Se utilizaron datos empíricos para investigar los factores que afectan la recuperación de las cadenas de suministro ante disrupciones, como la colaboración, la visibilidad, la flexibilidad, la orientación analítica y la gestión de riesgos. Se realizó una revisión de la literatura para elaborar el cuestionario que se aplicó a las industrias manufactureras en Brasil. Los métodos estadísticos utilizados incluyen el análisis factorial confirmatorio y el modelado de ecuaciones estructurales. Los resultados indican que el paquete de capabilidades de resiliencia -colaboración, flexibilidad, visibilidad y orientación analítica- afecta positivamente la resiliencia de la cadena de suministro. Por lo tanto, mejorar estas capabilidades permitirá que las cadenas de suministro se recuperen más adecuadamente de las disrupciones. También se descubrió que la gestión de riesgos por sí sola no favorece la recuperación de las disrupciones. Además, existe un impacto mutuo entre las capacidades de resiliencia y la gestión de riesgos.

PALABRAS CLAVE I Resiliencia de la cadena de suministro, capabilidades de resiliencia, gestión de riesgos, recuperación, modelos de ecuaciones estructurales. 


\section{INTRODUÇÃO}

A pandemia da COVID-19, que está impactando organizações e suas cadeias de suprimento em todo o mundo (Cosgrove, 2020); a saída do Reino Unido da União Europeia, que levou a Domino’s Pizza a gastar US\$ 8,5 milhões em estoque de ingredientes para evitar a interrupção em sua cadeia de suprimento (CNN, 2019); e o incêndio na fábrica da Phillips, que afetou a produção da Ericsson (Norrman \& Jansson, 2004), são exemplos de como riscos e incertezas podem ser devastadores, não apenas para uma organização, mas também para todas as demais que compõem sua cadeia de suprimentos (Ambulkar, Blackhurst \& Grawe, 2015) .

Gestores e autores têm enfatizado a necessidade de construir cadeias de suprimento resilientes para promover a rápida recuperação após interrupções (Brusset \& Teller, 2017; Pettit, Croxton \& Fiksel, 2019). É essencial que se construa cadeias resilientes dada a interdependência de seus membros, com as organizações impactando e sendo impactadas por interrupções internas ou externas. Como membros de cadeias de suprimento, as organizações devem desenvolver suas capabilidades para se tornarem resilientes a fim de contribuir com o retorno às operações normais ou aprimoradas o mais rápido possível (Jüttner \& Maklan, 2011; Kwak, Seo \& Mason, 2018; Pettit et al., 2019).

Algumas das capabilidades de resiliência mais amplamente discutidas na literatura até o ano de 2016 foram a colaboração (Jüttner \& Maklan, 2011; Pettit, Fiksel \& Croxton, 2010; Scholten \& Schilder ., 2015), flexibilidade (Jüttner \& Maklan, 2011; Ponomarov \& Holcomb, 2009; Sheffi \& Rice, 2005), visibilidade (Brandon-Jones, Squire, Autry \& Petersen, 2014; Christopher \& Peck, 2004; Scholten, Scott \& Fynes, 2014; Wieland \& Wallenburg, 2013) e, mais recentemente, orientação analítica (Dubey et al., 2019; Ivanov, Dolgui, Das \& Sokolov, 2019; Oliveira \& Handfield, 2019; Papadopoulos et al., 2017; Xu, Zhang, Feng \& Yang, 2020).

Outros estudos quantitativos investigaram a relação entre essas capabilidades de resiliência e a resiliência da cadeia de suprimentos individualmente: (i) flexibilidade (Brusset \& Teller, 2017; Mandal, Sarathy, Korasiga, Bhattacharya \& Dastidar, 2016), (ii) colaboração (Kumar \& Anbanandam, 2019; Mandal et al., 2016), (iii) visibilidade (Brandon-Jones et al., 2014; Kumar \& Anbanandam, 2019; Mandal et al., 2016) e orientação analítica (Dubey et al., 2019; Singh e Singh, 2019). Não discordamos de que cada capabilidade tem uma influência positiva na resiliência da cadeia de suprimento. 0 principal argumento, porém, é que essas capabilidades devem agir em sinergia e serem reunidas em que denominamos de "pacote de capabilidades", indispensável e aplicável em toda cadeia de suprimento. Esse argumento está de acordo com Daugherty et al. (2006) e Scholten e Schilder (2015), uma vez que cada capabilidade isoladamente é insuficiente e pode 'subotimizar' os resultados de resiliência da cadeia de suprimentos.

Este estudo apresenta um avanço em relação ao modelo teórico inicialmente sugerido por Alvarenga, Oliveira, Zanquetto-Filho e Santos (2018a), propondo que existe um impacto mútuo entre o pacote de capabilidades de resiliência e a gestão de risco da cadeia de suprimentos, e que esses construtos, por sua vez, tenham efeito na resiliência dessa cadeia. A pesquisa, portanto, visa contribuir ampliando o conhecimento sobre a resiliência da cadeia de suprimentos e seus antecedentes a partir de um ciclo de avanços conceituais, testes empíricos e novos avanços conceituais (Colquitt \& Zapata-Phelan, 2007).

Em teoria, embora a gestão de risco da cadeia de suprimento seja importante para sua resiliência, nossa hipótese principal é de que o pacote de capabilidades tem um maior impacto positivo nesse sentido. Acreditando nesse argumento lógico e conceitual, propomos um modelo teórico e o testamos empiricamente.

Do ponto de vista prático, caso a hipótese for verdadeira, as cadeias de suprimento devem gerenciar suas capabilidades de resiliência de forma integrada, como um pacote, e não as aplicar separadamente. Considerando 
o cenário atual e futuro, no qual os gestores acreditam que as interrupções serão cada vez mais frequentes, isso será fundamental para ampliar o desempenho da cadeia de suprimentos.

\section{CONSTRUÇÃO TEÓRICA DA HIPÓTESE}

\section{Resiliência em cadeia de suprimentos}

Não existe consenso sobre a definição de resiliência da cadeia de suprimentos, conforme evidenciado pelos trabalhos de Ribeiro e Barbosa-Póvoa (2018) e Wong, Lirn, Yang e Shang (2019). As visões variam desde aquelas que consideram a resiliência da cadeia de suprimentos como a forma de lidar com uma interrupção e os momentos subsequentes, até aquelas que também consideram o momento antes de uma interrupção (Ali, Mahfouz \& Arisha, 2017). A definição que adotamos é a da capabilidade que a cadeia apresenta de se recuperar ou passar para um estado mais desejável após a ocorrência de uma interrupção (Brandon-Jones et al., 2014; Christopher \& Peck, 2004; Wong et al., 2019). Assim, consideramos que resiliência é recuperação. 0 pacote de capabilidades proposto permite o retorno mais rápido às operações (Brandon-Jones et al., 2014) ou a recuperação após um evento de risco inesperado (Kwak et al., 2018). Portanto, adaptamos os indicadores de Graeml e Peinado (2014) e Brandon-Jones et al. (2014) usados para avaliar a resiliência da cadeia de suprimentos.

De acordo com Pettit, Fiksel e Croxton (2010), as cadeias de suprimento devem enfrentar vulnerabilidades desenvolvendo capabilidades de resiliência, que são definidas como os "atributos que permitem à empresa se antecipar ou se recuperar de interrupções" (Pettit et al., 2010, p. 6, tradução nossa). As capabilidades que aparecem com mais frequência na literatura sobre resiliência da cadeia de suprimentos são colaboração (Jüttner \& Maklan, 2011; Pettit et al., 2010; Scholten \& Schilder, 2015), flexibilidade (Jüttner \& Maklan, 2011; Ponomarov \& Holcomb, 2009; Sheffi \& Rice, 2005) e visibilidade (Brandon-Jones et al., 2014; Christopher \& Peck, 2004; Scholten et al., 2014; Wieland \& Wallenburg, 2013).

\section{Pacote de capabilidades de resiliência}

Detalhamos aqui as capabilidades inclusas no pacote proposto, destacando a orientação analítica como uma capabilidade de resiliência particular. Várias capabilidades foram selecionadas usando a metodologia construtivista do processo de desenvolvimento do conhecimento (Proknow-C) (Lacerda, Ensslin \& Ensslin, 2012; Marafon, Ensslin, Lacerda \& Ensslin, 2015). Com base em 72 artigos indexados na plataforma Web of Science ou publicados na Ebsco, Science Direct e Emerald nos últimos 30 anos, com títulos contendo os termos, em inglês, "supply chain resilience" (resiliência da cadeia de suprimentos) ou "resilient supply chain" (cadeia de suprimentos resiliente), identificamos os artigos mais frequentemente citados (21 artigos), respondendo por 90\% das citações sobre o tema. Esses artigos e outros mais atuais compativeis com o tema foram utilizados para a confecção da base do modelo inicial.

\section{Colaboração}

Colaboração é definida como o relacionamento que existe entre os membros principais da cadeia de suprimento e que permite estratégias conjuntas, troca de informações e compartilhamento de conhecimento com seus principais compradores e fornecedores (Barratt, 2004; Cao \& Zhang, 2011).

O estudo empírico de Jüttner e Maklan (2011) revelou que a colaboração entre os membros da cadeia mitiga os impactos negativos das interrupções. Além disso, a comunicação e a cooperação entre eles têm impacto na 
resiliência de toda cadeia (Wieland \& Wallenburg, 2013) tanto estatística quanto significativamente. A pesquisa quantitativa permitiu a Kumar e Anbanandam (2019) e Mandal et al. (2016) estabelecer que a colaboração também causa impacto na resiliência da cadeia.

As vantagens de elementos como a colaboração para a construção de uma cadeia de suprimentos resiliente tornam-se claras durante eventos como, por exemplo, o colapso financeiro da transportadora Hanjin Shipping, em 2017, que teve seus navios impedidos de entrar em portos ao redor do mundo e abandonou US\$ 14 bilhões em mercadorias. Na época, a AGL Logistics e sua rede de parceiros encontrou espaço para as remessas dos seus clientes com outra transportadora, que honrou a taxa original cobrada pela Hanjin. Sem esse suporte a empresa, teria que esperar meses pela liberação para atracar nos Estados Unidos (Roberson, 2019).

\section{Flexibilidade}

A flexibilidade da cadeia de suprimentos está relacionada à capacidade dos membros de ajustar seus processos-chave, tanto internos quanto orientados ao cliente, reagindo ou se adaptando à dinâmica ambiental e, consequentemente, entregando valor aos clientes e garantindo a lucratividade da cadeia (Merschmann \& Thonemann, 2011; Swafford, Ghosh \& Murthy, 2006, 2008). O construto nesta pesquisa é operacionalizado como uma capabilidade de reação/adaptação no fornecimento e entrega (Swafford et al., 2008).

A flexibilidade de uma cadeia permite uma resposta rápida quando ocorrem mudanças no fornecimento e reposição - como uma redução no tempo de desenvolvimento do produto, por exemplo - para então criar uma vantagem competitiva baseada na capacidade da cadeia de lidar com os recursos disponíveis e sua adaptabilidade (Sánchez \& Pérez, 2005; Merschmann \& Thonemann, 2011; Thomé, Scavarda, Pires, Ceryno \& Klingebiel, 2014). O plano do CEO da H\&M, Karl-Johan Persson, de melhorar os resultados de desempenho passando da moda rápida para o varejo inteligente, significou que o varejista teve que construir resiliência operacional criando uma cadeia de suprimentos mais rápida, flexível e baseada em tecnologia (Lopez, 2018).

A flexibilidade promove resiliência (Ojha, Ghadge, Tiwari e Bititci, 2018). Para ser considerada resiliente, a cadeia deve desenvolver a flexibilidade necessária para mudar suas entradas ou saídas rapidamente, ou reconhecer como fazer essas mudanças. Ela pode ser desenvolvida, entre outras coisas, cultivando múltiplas fontes de suprimento e reposição; desenvolvendo flexibilidade de contrato; promovendo o compartilhamento de riscos e melhorando a gestão de estoque (Pettit et al., 2010). Um estudo do setor automotivo brasileiro por Scavarda, Ceryno, Pires e Klingebiel (2015) também confirmou que a flexibilidade resulta em resiliência.

\section{Visibilidade}

Visibilidade é a capabilidade da empresa focal de visualizar toda a cadeia de suprimento, a consciência situacional da atividade e as condições ambientais em que opera. Mais especificamente, uma empresa pode observar as condições de estoque de clientes e fornecedores (Christopher \& Peck, 2004) e evitar, por exemplo, "efeitos-chicote" mais severos.

Com base na perspectiva da empresa focal, a visibilidade da cadeia pode ser determinada como uma capabilidade mensurável e que é influenciada pela quantidade e qualidade das informações trocadas. Isso a favorece, pois, permite visualizar com precisão os níveis de estoque e as demandas de seus principais clientes e fornecedores (Barratt \& Oke, 2007; Brandon-Jones et al., 2014; Caridi, Crippa, Perego, Sianesi \& Tumino, 2010; 
Caridi, Perego e Tumino, 2013). As medidas de visibilidade aqui adotadas baseiam-se nos indicadores desenvolvidos por Brandon-Jones et al. (2014).

Visibilidade também envolve uma associação positiva com agilidade, ou o quão rápido a cadeia pode se recuperar de interrupções (Brandon-Jones et al., 2014; Christopher \& Peck, 2004; Namdar, Li, Sawhney \& Pradhan, 2018). Primeiramente, Brandon-Jones et al. (2014) verificaram quantitativamente o impacto positivo da visibilidade na resiliência da cadeia de suprimentos. Posteriormente, Kumar e Anbanandam (2019) e Mandal et al. (2016) enfatizaram que a visibilidade impacta a resiliência da cadeia de suprimento.

\section{Orientação Analítica}

Conforme observado por Ladeira et al. (2016), a abordagem analítica envolve "a utilização extensiva de dados críticos e modelos explicativos e preditivos, bem como a gestão baseada em fatos para orientar decisões e ações gerenciais" (p. 487). Os membros de cadeias com orientação analítica processam dados de forma eficiente por meio de análises quantitativas para tomar decisões sobre seus processos baseadas em fatos (Cao, Duan \& Li, 2015; Souza, 2014).

Trkman, Mccormack, Oliveira e Ladeira (2010) verificaram empiricamente que a abordagem analítica aos processos críticos de uma cadeia de suprimentos resulta em um melhor desempenho. Chae, Olson e Sheu (2014) também observaram que as cadeias analíticas têm impacto no desempenho dos membros da cadeia. Mais especificamente, os dados podem ser usados para produzir insights sobre o contexto da tomada de decisão organizacional (Cao et al., 2015), o que favorece a resiliência pela prevenção, adaptação e tomada de decisão eficiente para lidar com interrupções. Um exemplo é a Healthcare Ready, organização que se concentra em ajudar a indústria farmacêutica a responder a desastres naturais, está usando tecnologia de rastreamento de estoque no apoio a análises preditivas para fabricantes e distribuidores em seus preparativos no trato de diferentes tipos de desastres (Wollenhaupt, 2019).

As definições de orientação analítica em cadeias de suprimentos estão intimamente relacionadas à capabilidade de coletar e analisar informações e eventos em ambientes internos e externos. Os gêmeos digitais, por exemplo, são representações virtuais de objetos projetados para otimizar a operação de ativos e têm um forte potencial em contribuir para que as cadeias de suprimentos melhorem sua resiliência organizacional (“Gartner highlights ‘digital twins' as an emerging organizational resilience tool”, 2018; Ivanov et al., 2019).

Entende-se, portanto, que a geração, processamento, armazenamento, recuperação, compartilhamento e aplicação de conhecimento para a tomada de decisão no que diz respeito à resiliência são potencializados pelo uso de uma abordagem analítica (Barbosa \& Vicente, 2018; Dubey et al., 2019; Ivanov et al., 2019). Consequentemente, o uso desse tipo de abordagem pode ajudar na recuperação em casos de interrupção (Dubey et al., 2019; Papadopoulos et al., 2017).

É digno de nota que melhorar o conjunto de capabilidades permite maior resiliência, mesmo quando se considera o impacto de uma delas. Entretanto, até onde entendemos, o impacto dessas capabilidades aplicados em conjunto nunca foi testado na literatura antes. Com base nesses argumentos, nossa primeira hipótese de pesquisa é:

H1: 0 pacote de capabilidades de resiliência tem um impacto positivo na resiliência da cadeia de suprimentos. 


\section{Gestão de risco da cadeia de suprimentos}

A gestão de risco da cadeia de suprimento (GRCS) envolve a identificação, avaliação e controle dos riscos internos e externos que podem afetar o desempenho de uma cadeia, a fim de eliminar ou reduzir a probabilidade ou impacto de eventos que venham a interromper as operações de seus membros (Jüttner, Peck \& Christopher, 2003; Sodhi, Son \& Tang, 2012). Assumimos que os riscos devem ser gerenciados em conjunto com os membros de uma cadeia, e que uma GRCS eficiente identifica, avalia, controla e monitora riscos (Hallikas, Karvonen, Pulkkinen, Virolainen \& Tuominen, 2004; Tummala \& Schoenherr, 2011).

Estudos recentes indicam que existe uma relação entre GRCS e a resiliência de uma cadeia (Pavlov, Ivanov, Dolgui \& Sokolov, 2018; Rajesh, 2017; Sáenz, Revilla \& Acero, 2018), visto que as estratégias de GRCS correm paralelamente aos objetivos de resiliência (Namdar et al., 2018; Zineb, Brahim e Houdaifa, 2017). Ainda assim, Jüttner e Maklan (2011) argumentam que a gestão de risco oferece conhecimento de risco ambiental, aumentando assim a resiliência. Da mesma forma, Colicchia, Dallari e Melacini (2010) verificaram que a adoção de estratégias de gestão de risco eficientes para lidar com a variabilidade do ciclo (lead time) também aumenta a resiliência da cadeia.

Se executada corretamente, a GRCS não só reduz as vulnerabilidades que podem afetar as operações da organização como consequência de interrupções na cadeia, mas também prepara a organização para mitigar quaisquer riscos possíveis (Norrman \& Jansson, 2004). Embora esse estudo presuma que uma cadeia de suprimentos pode se recuperar sem necessidade de prevenção (já que se concentra na recuperação como uma extensão da resiliência), a literatura científica descobriu que a GRCS pode ter um impacto na resiliência da cadeia de suprimentos. Isso envolve principalmente a prevenção de eventos que possam interromper suas operações, enquanto permite o desenvolvimento de ações que podem restaurá-las (Ceryno, Scavarda, Klingebiel \& Yüzgülec, 2013; Colicchia et al., 2010; Jüttner \& Maklan, 2011).

H2: A gestão de risco da cadeia de suprimento tem um impacto positivo na sua resiliência.

\section{Relacionamento entre o pacote de capabilidades de resiliência e a gestão de risco da cadeia de suprimentos}

O gerenciamento de risco também é influenciado pelas capabilidades que abordamos nas seções anteriores. 0 gerenciamento eficiente de riscos da cadeia de suprimentos envolve gerenciamento colaborativo, flexibilidade, visibilidade e orientação analítica. Li, Fan, Lee e Cheng (2015) mostraram que a duração do relacionamento e a confiança fortalecem a eficácia do compartilhamento de informações de risco. Christopher e Peck (2004) apontaram que a troca de informações entre os membros da cadeia de suprimentos é uma prioridade chave para reduzir o risco. Mais exemplos da relação entre colaboração e GRCS, podem ser encontrados nas obras de Hernandez e Haddud (2018), Zineb et al. (2017), Prakash, Gautam e Soni (2018) e Durach e Machuca (2018). Estudos mostraram que uma maior visibilidade promove uma melhor compreensão dos riscos e de como mitigá-los (Nooraie \& Parast, 2015; Prakash et al., 2018; Urciuoli \& Hintsa, 2018). A flexibilidade pode ser usada para mitigar ou evitar impactos de risco, modificando entradas e saídas (Prakash et al., 2018; Sheffi \& Rice, 2005).

O efeito oposto também pode ser identificado em Jüttner e Maklan (2011) que concluíram, por meio de um estudo de caso longitudinal com três cadeias de suprimentos, que as ações orientadas em relação ao efeito do 
risco - considerando a partilha de riscos e os riscos de cobertura por meio de recursos redundantes - têm um impacto positivo nas capabilidades de resiliência em termos de flexibilidade, visibilidade e colaboração. A GRCS ajuda a reduzir o comportamento oportunista, permite a dispersão das perdas, desenvolve a confiança, permite que as interrupções sejam absorvidas pelas partes e reduz os pipelines operacionais de suprimento, reduzindo assim os ciclos da cadeia (lead times) (Jüttner \& Maklan, 2011).

Especificamente em relação a GRCS e às capabilidades analíticas, Tummala e Schoenherr (2011) argumentam que o gerenciamento de dados permite a coleta, armazenamento e recuperação de informações de risco e, consequentemente, melhora o GRCS como um todo. Tang e Musa (2011) relatam que uma abordagem quantitativa para gerenciar os riscos da cadeia de suprimentos precisa ser desenvolvida. Por um lado, a cadeia deve desenvolver um pacote de capabilidades, conforme proposto neste estudo, para identificar, avaliar e propor estratégias de mitigação para a gestão adequada de riscos (Alvarenga, Oliveira, Zanquetto-FiIho, \& Santos, 2018b), que inclui a necessidade de uma orientação analítica para identificar e mensurar riscos de forma eficiente, facilitando o gerenciamento adequado (Ittmann, 2015; Pettit et al., 2019). Por outro lado, a GRCS pode oferecer aos membros da cadeia de suprimentos dados úteis e melhorar sua orientação analítica.

Apenas os relacionamentos individuais dessas capabilidades e a GRCS foram testados na literatura. Como o enfoque aqui é no pacote dessas capabilidades, não no impacto individual, a terceira hipótese da pesquisa é:

H3: Existe uma relação positiva mútua entre o pacote de capabilidades de resiliência e a gestão de risco da cadeia de suprimento.

\section{METODOLOGIA}

Os dados foram coletados nos meses de agosto e dezembro de 2017, por meio de questionário online de três blocos, apresentado de forma aleatória, em escala tipo Likert de sete pontos, sendo um ponto (discordo totalmente) a sete pontos (concordo totalmente). Depois de desenvolvido, o questionário foi testado com dez gerentes de fábrica ou da cadeia de suprimentos de empresas de manufatura. 0 instrumento foi então enviado a todas as indústrias de transformação pertencentes as federações industriais de Minas Gerais, São Paulo, Rio de Janeiro e do Espírito Santo. As empresas de Minas Gerais foram acessadas por meio do Centro Industrial e Empresarial de Minas Gerais (CIEMG), enquanto que as demais por meio da Editora Brasileira de Guias Especiais (EBGE).

A amostra final ficou composta por 143 respostas completas de 151 recebidas no total. Usando o software $G^{\star}$ Power, foi calculado o tamanho da amostra necessário com base em dois construtos preditores (o pacote de capabilidades de resiliência e a gestão de risco), definindo o,15 como o tamanho do efeito, um poder de teste de 0,80 e 0,05 como a probabilidade de erro. 0 tamanho mínimo da amostra encontrada foi de 43 casos. 0 mesmo software foi utilizado post hoc para calcular o poder ideal do teste dado o tamanho da amostra obtido, e atingiu um poder explicativo de $99,84 \%$.

A análise da modelagem de equações estruturais foi processada usando o software SmartPLS (Ringle, Wende \& Becker, 2014). De acordo com Hair et al. (2009), a modelagem de equações estruturais fornece a possibilidade de estimar com eficiência uma série de equações de regressão múltiplas separadas, que podem ser calculadas simultaneamente, considerando as relações entre as variáveis manifestadas e seus construtos. 


\section{ANÁLISE DE DADOS E RESULTADOS}

\section{Descrição da amostra}

O número de funcionários das empresas respondentes foi utilizado para indicar o tamanho das empresas, pois, a utilização da receita bruta para esse fim no Brasil é uma medida mais complexa. Organizações com até 19 funcionários foram consideradas microempresas, aquelas com 19 a 99 funcionários foram consideradas pequenas, com 100 a 499 funcionários foram consideradas médias, enquanto as empresas com mais de 499 funcionários foram consideradas grandes (Tabela 1). De acordo com o anuário do Serviço Brasileiro de Apoio às Micro e Pequenas Empresas (SEBRAE, 2016) , 98\% das empresas brasileiras na indústria de transformação são micro ou pequenas. Na amostra, $77 \%$ dos respondentes são micro/pequenas empresas e de médio porte, portanto, foi realizado um teste de heterogeneidade observando o porte das empresas com vistas a melhorar a confiabilidade da amostra e a consistência dos resultados.

Tabela 1. Descrição da amostra

\begin{tabular}{c|c|c|c|c|c|c|c|c}
\hline \multicolumn{2}{c|}{ Quantidade de funcionários } & \multicolumn{2}{c|}{ Cargos dos respondentes } & \multicolumn{3}{c}{ Principal função } \\
\hline Categoria & Quantidade & $\%$ & Categoria & Quantidade & $\%$ & Categoria & Quantidade & $\%$ \\
\hline 19 & 54 & 37,80 & Presidentes & 19 & 13,30 & Produção & 62 & 43 \\
\hline $19-99$ & 56 & 39,20 & Diretoria & 46 & 32,20 & Compras & 34 & 24 \\
\hline $100-499$ & 28 & 19,6 & Gerentes & 58 & 40,60 & $\begin{array}{c}\text { Marketing/ } \\
\text { Vendas }\end{array}$ & 17 & 12 \\
\hline$>500$ & 5 & 3,5 & Assistentes & 8 & 5,60 & Outros & 30 & 21 \\
\hline & & & Outros & 12 & 8,40 & & & \\
\hline
\end{tabular}

\section{Modelo de mensuração}

Uma análise da confiabilidade interna dos construtos revelou que os valores de confiabilidade do composto estavam de acordo com os limites estipulados por Hair, Hult, Ringle e Sarstedt (2017), que é maior que 0,7 e menor que 0,95 . Em relação à validade convergente - ou como os indicadores se correlacionam com os demais do mesmo construto (Hair et al., 2017) - as cargas externas obtiveram valores superiores a 0,703, exceto para a variável R3. Embora essa variável de carga externa esteja abaixo do ponto de corte, ela foi considerada aceitável (acima de 0,4 ) por sua significância estatística, sendo que sua exclusão não garantiria uma melhora substancial no modelo de mensuração. A variância média extraída (AVE) também apresentou valores aceitáveis, maiores que 0,5 . Os resultados do questionário e da mensuração são mostrados na Tabela 2 abaixo.

\section{Tabela 2. Questionário e resultados da mensuração}

Em relação à sua cadeia de suprimentos (organização, principais clientes e principais fornecedores):

Orientação analítica - confiabilidade composta $=0,87$, variância média extraída $=0,70$.

A1 - A análise quantitativa dos dados direciona as ações gerenciais. (Carga - 0,767)

A2 - Os membros da cadeia utilizam o conhecimento gerado a partir da análise quantitativa dos dados para aprimorar os processos da cadeia. (Carga - o,879) 
Em relação à sua cadeia de suprimentos (organização, principais clientes e principais fornecedores):

A3 - Para suportar as decisões gerenciais, os membros da cadeia são capazes de processar eficientemente os dados.(Carga - 0,866)

Colaboração - confiabilidade composta $=0,88$, variância média extraída $=0,71$.

C1 - As opiniões dos membros da cadeia são geralmente utilizadas para aprimorar os processos de suprimento. (Carga - 0,735)

C2 - Os membros da cadeia comunicam de forma efetiva mudanças em seus processos de suprimento . (Carga - 0,894)

C3 - Os membros da cadeia executam planos conjuntos de melhorias dos processos de suprimento. (Carga - 0,891)

Flexibilidade - confiabilidade composta $=0,84$, variância média extraída $=0,64$.

F1 - Quando necessário, os membros da cadeia conseguem modificar a capacidade de volume de produção. (Carga - 0,838)

F2 - Quando necessário, os membros da cadeia conseguem acomodar mudanças no mix de produção (variedade de produtos). (Carga - 0,835)

F3 - Quando necessário, os membros da cadeia conseguem reduzir o tempo de produção. (Carga - 0,733)

Visibilidade - confiabilidade composta $=0,83$, variância média extraída $=0,63$.

V1 - É possível visualizar com precisão as informações acerca dos níveis de demanda dos clientes. (Carga - 0,807)

V2 - É possível visualizar com precisão as informações acerca dos níveis de estoque dos clientes. (Carga - 0,856)

V3 - É possível visualizar com precisão as informações acerca dos níveis de estoque dos fornecedores. (Carga - 0,714)

Gerenciamento de risco da cadeia de suprimentos - confiabilidade composta $=0,92$, variância média extraída $=0,74$.

S1 - Possui processos formais para identificar riscos. (Carga - 0,799)

S2 - Consegue minimizar o impacto dos riscos. (Carga - 0,838)

S3 - Os riscos são frequentemente avaliados pelos membros da cadeia.(Carga - 0,919)

$\mathrm{S}_{4}$ - Os riscos são frequentemente monitorados pelos membros da cadeia. (Carga - 0,891)

Resiliência da cadeia de suprimentos - confiabilidade composta $=0,81$, variância média extraída $=0,60$.

R1 - Consegue retomar rapidamente o fluxo de materiais em caso de interrupções. (Carga - 0,845)

R2 - Consegue retornar facilmente ao seu estado original em caso de interrupções. (Carga - 0,856)

R3 - Consegue desenvolver conhecimento útil a partir de interrupções. (Carga - 0,603)

A validade discriminante foi verificada por meio da análise de Fornell-Lacker (Tabela 3). 0 objetivo foi verificar se os respondentes perceberam os construtos do estudo de forma diferente (Hair et al., 2017). Posteriormente, observamos que a raiz quadrada da AVE do construto é maior do que a correlação do construto com os outros do modelo. Os resultados demonstram que os construtos podem ser considerados únicos e cada construto está captando um fenômeno diferente.

Tabela 3. Análise de Fornell-Lacker

\begin{tabular}{l|c|c|c|c|c|c}
\hline Construto & Colaboração & Flexibilidade & GRCS & Orientação Analítica & RESCS & Visibilidade \\
\hline Colaboração & $\mathbf{0 , 8 4 3}$ & & & & \\
\hline Flexibilidade & 0,549 & $\mathbf{0 , 8 0 3}$ & & & \\
\hline GRCS & 0,458 & 0,325 & $\mathbf{0 , 8 6 3}$ & & \\
\hline $\begin{array}{l}\text { Orientação } \\
\text { Analítica }\end{array}$ & 0,570 & 0,358 & 0,556 & $\mathbf{0 , 8 3 9}$ & & \\
\hline RESCS & 0,350 & 0,379 & 0,294 & 0,133 & $\mathbf{0 , 7 7 7}$ & 0,231 \\
\hline Visibilidade & 0,304 & 0,192 & 0,263 & 0,341 & 0,794 \\
\hline
\end{tabular}




\section{Resultados do modelo hierárquico}

Os resultados do modelo após a execução de um procedimento de bootstrapping com 5.000 subamostras (Tabela 4) confirmaram estatisticamente que colaboração, flexibilidade, visibilidade e orientação analítica são componentes do pacote de capabilidades de resiliência, já que todos os valores de $p$ foram menores que 0,05 , corroborando teoricamente argumentos desenvolvidos anteriormente. Podemos observar que a capabilidade de colaboração tem demonstrado maior relevância na formação do construto pacote de capabilidades de resiliência, seguida da orientação analítica.

Tabela 4. Modelo de mensuração do construto de segunda ordem

\begin{tabular}{|c|c|c|c|c|}
\hline \multirow{3}{*}{$\begin{array}{l}\text { Variável latente (segunda } \\
\text { ordem) }\end{array}$} & \multirow{3}{*}{$\begin{array}{c}\text { Construto de primeira } \\
\text { ordem }\end{array}$} & \multirow{2}{*}{$\begin{array}{c}\text { Colinearidade } \\
\text { VIF }\end{array}$} & \multicolumn{2}{|c|}{$\begin{array}{l}\text { Significância e relevância dos } \\
\text { indicadores }\end{array}$} \\
\hline & & & Peso & Valor $p$ \\
\hline & & $<5$ & - & $<0,05$ \\
\hline \multirow{4}{*}{$\begin{array}{l}\text { Pacote de Capabilidades de } \\
\text { resiliência }\end{array}$} & Colaboração & 1,884 & 0,421 & $5,684 \mathrm{E}-14$ \\
\hline & Flexibilidade & 1,437 & 0,336 & $5,684 \mathrm{E}-14$ \\
\hline & Visibilidade & 1,155 & 0,234 & $5,684 \mathrm{E}-14$ \\
\hline & Orientação Analítica & 1,559 & 0,337 & $6,859 \mathrm{E}-08$ \\
\hline
\end{tabular}

Figura 1. Resultados do modelo proposto

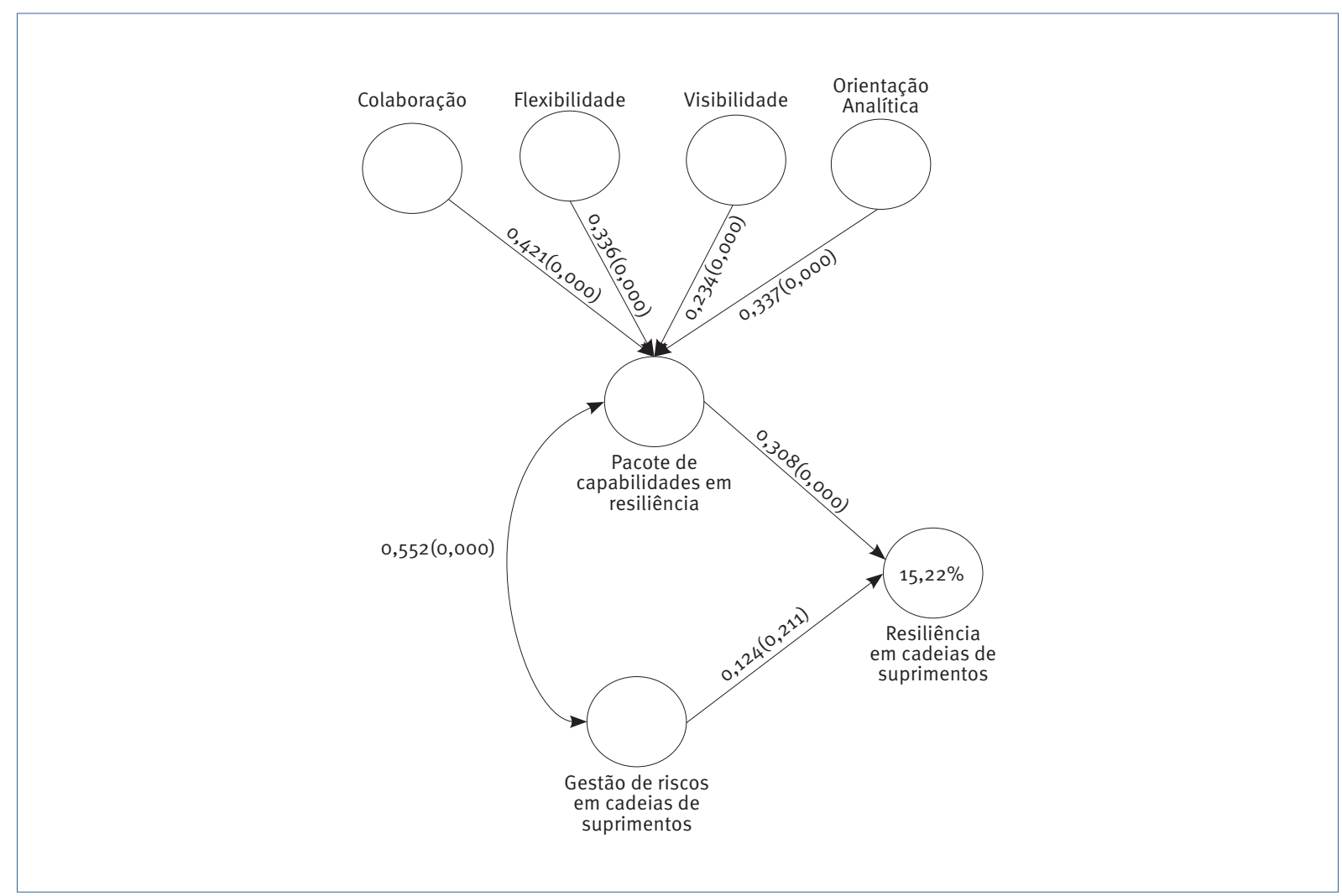




\section{Modelo estrutural}

O modelo estrutural foi avaliado pela análise dos resultados de cada regressão. Como não é possível testar uma seta dois sentidos no software SmartPLS, as relações mútuas foram determinadas testando o coeficiente de correlação de Pearson em relação aos escores das variáveis latentes. Depois de executar um procedimento de bootstrapping com 5.000 subamostras e o teste de correlação de Pearson, a Figura 1 ilustra os resultados do modelo, com os números entre parênteses delineando a relação a. Ressaltamos que não existe colinearidade entre os construtos preditivos, pois o fator de inflação da variância (VIF) foi de 1,455.

Os valores do coeficiente de caminho e a confirmam as hipóteses $\mathrm{H}_{1}$ e $\mathrm{H}_{3}$. A hipótese $\mathrm{H}_{2}$, no entanto, foi rejeitada. Os resultados também demonstram que $15,22 \%$ da variação na resiliência da cadeia de suprimento vem de variações no pacote de capabilidades de resiliência e na GRCS. Embora esse resultado possa ser interpretado como fraco, é significativo e relevante que existam muitos outros fatores além dos abordados aqui, sendo que esses fatores podem impacto na resiliência da cadeia de suprimentos.

\section{DISCUSSÃO}

\section{Implicações gerenciais}

Na prática, os resultados indicam que os membros da cadeia de suprimento devem buscar maior colaboração, visibilidade, flexibilidade e orientação analítica em seus processos se desejam evitar o colapso de suas operações na ocorrência de interrupções. Descobrimos que a GRCS tem um efeito sinérgico para com as capabilidades de resiliência sem, entretanto, afetar diretamente a resiliência em si. Em outras palavras, nosso argumento é que a GRCS ajuda na prevenção, mas quando se trata de um evento disruptivo, a solução é encontrada no pacote de capabilidades de resiliência, logo, os gerentes da cadeia de suprimentos precisam garantir a robustez dessas capabilidades. Como as capacidades de resiliência trabalham juntas, uma análise do nível dessas capabilidades pode mostrar aos gerentes onde investir para garantir melhores resultados de resiliência.

Para melhorar sua visibilidade, as organizações devem investir na obtenção de informações sobre eventos, recursos, produção, status do processo e planos futuros dos parceiros (Caridi et al., 2010). Promover a colaboração entre os membros da cadeia, portanto, é fundamental na obtenção de melhores resultados em termos de visibilidade (Holcomb, Ponomarov \& Manrodt, 2011). As informações obtidas devem ser úteis, confiáveis e recebidas no momento certo para a tomada de decisão (Barratt \& Oke, 2007; Brandon-Jones et al., 2014; Caridi, Moretto, Perego \& Tumino, 2014; Oliveira e Handfield, 2019).

As cadeias exigem que todos os dados gerados e obtidos por meio da colaboração entre os membros sejam processados para obtenção de maior visibilidade, transparência e informações em tempo real (Oliveira \& Handfield, 2019; Zhu, Song, Hazen, Lee \& Cegielski, 2016). Sugerimos, portanto, investir em sistemas e tecnologias de informação que possibilitem o compartilhamento e processamento adequado da informação (Barratt \& Oke, 2007; Brandon-Jones et al., 2014; Caridi et al., 2014). Como apontado por Kirchoff (2019, p.1, tradução nossa), "novas tecnologias como o blockchain trazem uma promessa de conectar melhor os membros da cadeia de suprimentos e aprimorar a confiança e a transparência".

Para melhorar a colaboração, a empresa focal e seus principais clientes e fornecedores devem desenvolver fatores-chave, como troca de informações, confiança, interações pessoais e planejamento conjunto (Kohli \& Jensen, 2010). 0 investimento em tecnologias como as mencionadas acima pode promover um maior nível 
de integração da cadeia, resultando em novas oportunidades de melhoria de processos (Bronzo et al., 2013; Vanpoucke, Vereecke \& Wetzels, 2014).

Uma orientação analítica facilita a identificação de mudanças no comportamento do consumidor, oportunidades de desenvolvimento de novos produtos e novos mercados e a absorção de informações externas (Teo, Nishant \& Koh, 2016); em outras palavras, maior flexibilidade. Visando aprimorar sua orientação analítica, as cadeias devem ter à disposição os sistemas de apoio à decisão certos, no momento certo, para que pessoas qualificadas possam aproveitar os dados produzidos (Davenport, Harris, Long \& Jacobson, 2001; Laursen \& Thorlund, 2010). Como Srinivasan e Swink (2018) apontaram, visibilidade e flexibilidade são complementares à análise. A visibilidade promove dados para análises, e os insights gerados por meio de análises só podem ser colocados em prática adequadamente se os membros da cadeia de suprimentos tiverem algum grau de flexibilidade (Srinivasan \& Swink, 2018).

Como nem todos os riscos podem ser evitados, os resultados da pesquisa não sugerem que as organizações devam adotar uma postura reativa à gestão de riscos, mas que apontem soluções quando eventos não identificados ou não gerenciados adequadamente resultem em interrupções. Portanto, as etapas de gestão de risco de (1) identificação de risco, (2) avaliação de risco, (3) controle de risco, 4) monitoramento de risco (Aqlan \& Lam, 2015; Tummala \& Schoenherr, 2011) ainda são vitais para a sobrevivência e prosperidade das cadeias de suprimento.

\section{Implicações teóricas}

Primeiramente, este estudo questionou os componentes da resiliência, tanto teórica quanto empiricamente. Verificou-se empiricamente que os membros da cadeia de suprimentos evitam interrupções por meio da gestão de riscos, mas não são necessariamente eficazes na recuperação de forma independente (resiliência). Assim, o estudo não confirmou o impacto direto da GRCS na resiliência. Portanto, a pesquisa contesta a afirmação de que a prevenção, que teoricamente se relaciona com a gestão de riscos, não é uma extensão ou um antecedente direto da resiliência da cadeia de suprimentos.

Além disso, todas as capabilidades em resiliência pesquisadas foram agrupadas em um pacote, uma vez que sua combinação aumenta a resiliência da cadeia de suprimentos. Até onde sabemos, isso nunca foi testado na literatura até agora. Como argumentamos nas implicações gerenciais, uma capabilidade é complementar a outra, o que significa que o poder total de sua eficiência só pode ser alcançado desenvolvendo-as em conjunto.

A contribuição teórica primária deste estudo envolveu o desenvolvimento e teste de um modelo teórico que inclui orientação analítica como um componente do pacote de capabilidades de resiliência. Isso também nunca foi testado anteriormente e, junto com as outras capabilidades propostas, provou explicar a resiliência da cadeia de suprimentos das indústrias de manufatura no sudeste do Brasil. 0 impacto individual da análise na resiliência da cadeia de suprimentos já foi tratado anteriormente em Dubey et al. (2019) e Singh e Singh (2019).

\section{CONCLUSÕES}

Com base no fato de que os riscos e incertezas da cadeia de suprimentos podem causar interrupções devastadoras para seus membros, este artigo teve como objetivo apresentar evidências empíricas que podem ser usadas para direcionar esforços com vistas a melhoria na resiliência da cadeia de suprimentos ao enfrentar eventos disruptivos e ameaças globais, como a pandemia da COVID-19. Argumentamos que, para serem resilientes, as cadeias de suprimento devem empreender esforços para desenvolver um pacote de capabilidades de resiliência, que inclui colaboração, flexibilidade, visibilidade e uma orientação analítica. 
Por meio de um estudo empírico sobre empresas de manufatura no Brasil, este artigo aborda um tópico relevante da gestão da cadeia de suprimentos. Ele analisa o impacto de um pacote de capabilidades de resiliência sobre a cadeia de suprimentos, em vez da influência de cada capabilidade, de forma isolada. Usando um método estatístico, incluindo análise fatorial confirmatória e modelagem de equações estruturais, foi possível testar as hipóteses da pesquisa. Em primeiro lugar, o estudo apresentou um pacote de capabilidades que foi validado pelos resultados do modelo hierárquico, incluindo orientação analítica como parte desse pacote. Em segundo lugar, os resultados do modelo estrutural permitiram confirmar a hipótese $\mathrm{H} 1$ que afirmava que o pacote de capabilidades de resiliência tem um impacto positivo na resiliência da cadeia de suprimento; e a hipótese $\mathrm{H}_{3}$, que dizia que existe uma relação mútua positiva entre o pacote de capabilidades de resiliência e a gestão de risco da cadeia de suprimento. Finalmente, os resultados levaram a refutar a hipótese $\mathrm{H} 2$, de que a gestão de risco da cadeia de suprimento tem um impacto positivo na sua resiliência. Os resultados das hipóteses possibilitaram validar o modelo teórico proposto por meio de um teste empírico.

Mais especificamente, nossa pesquisa oferece uma melhor interpretação do construto de resiliência da cadeia de suprimento, bem como observou se a colaboração, flexibilidade, visibilidade e orientação analítica são componentes de um pacote de capabilidades de resiliência. 0 estudo também confirmou não apenas se este pacote influencia a GRCS, mas também se a GRCS também o influencia. Os resultados indicaram que o pacote resulta em uma maneira mais fácil e rápida para as cadeias de suprimento retornarem à sua condição anterior após a ocorrência de interrupções, ou para evoluir para uma condição melhor e mais desejável; em outras palavras, cadeias de suprimento colaborativas, flexíveis, visíveis e analíticas são mais resilientes, justamente por contarem com essas capabilidades. A ausência delas leva a uma subotimização do resultado.

Os resultados ainda sugerem que a GRCS não afeta diretamente a resiliência da cadeia de suprimento. Uma explicação teórica para esse achado é que a prevenção não resolve as interrupções em uma empresa que precisa de ajuda para se recuperar. Alternativamente, as organizações podem desenvolver em conjunto capabilidades como colaboração, visibilidade, flexibilidade e orientação analítica, mas isso é teoricamente duvidoso, uma vez que outros estudos sugerem a prevenção como uma dimensão da resiliência em cadeias de suprimentos (Pavlov et al., 2018; Rajesh, 2017; Sáenz et al., 2018). Consequentemente, surge uma questão a partir deste estudo: A prevenção pode tornar as empresas mais resilientes ou a prevenção é uma condição necessária, porém, insuficiente para a resiliência?

Como estamos atualmente passando por uma interrupção global sem precedentes relacionada a COVID19, estudos futuros podem investigar quais capabilidades adicionais podem ter ajudado a minimizar o impacto do vírus nas cadeias de suprimento; em outras palavras, quais são as diferenças entre as cadeias de suprimento que foram mais ou menos impactadas? Estudos futuros também podem buscar lições na memória das cadeias de suprimento, que podem ser usadas para evitar ou recuperar-se de uma futura crise global.

É importante validar o modelo apresentado em outros contextos, cultura e ambientes, fora do Brasil. Também pode ser estimulante investigar como a estrutura da cadeia de suprimento impacta nas capabilidades, por exemplo, como a colaboração pode ser desenvolvida sob diferentes arranjos estruturais da cadeia de suprimento e seu impacto nos resultados de resiliência.

Por fim, sugerimos que pesquisas futuras investiguem outras capabilidades que também possam explicar a recuperação da cadeia de suprimentos, como proteção e segurança. Embora a "digitalização" das cadeias possa resultar em inúmeros benefícios, como foi argumentado ao longo do artigo, esse mesmo processo também apresenta outros riscos. Nesse sentido, as preocupações com a segurança cibernética estão emergindo como um tópico de pesquisa cada vez mais relevante no campo da resiliência da cadeia de suprimento. 


\section{AGRADECIMENTOS}

O presente trabalho foi realizado com apoio da Coordenação de Aperfeiçoamento de Pessoal de Nível Superior Brasil (CAPES) - Código de Financiamento 001.

\section{REFERÊNCIAS}

Ali, A., Mahfouz, A., \& Arisha, A. (2017). Analysing supply chain resilience: Integrating the constructs in a concept mapping framework via a systematic literature review. Supply Chain Management: An International Journal, 22(1), 16-39. doi: 10.1108/SCM-06-2016-0197

Alvarenga, M. Z., Oliveira, M. P. V. de, Zanquetto-Filho, H., \& Santos, W. R. dos. (2018a, December). Analytical supply chains: Are They more resilient? A model's proposition. Journal of Operations and Supply Chain Management, 11(2), 46-58. doi: 10.1266o/joscmv11n2p46-58

Alvarenga, M. Z., Oliveira, M. P. V. de, Zanquetto-Filho, H., \& Santos, W. R. dos. (2018b, December). Do analyticallyoriented supply chains better manage risks? Journal of Operations and Supply Chain Management, 11(2), 32-45. doi: 10.1266o/joscmv11n2p32-45

Ambulkar, S., Blackhurst, J., \& Grawe, S. (2015). Firm's resilience to supply chain disruptions: Scale development and empirical examination. Journal of Operations Management, 33-34, 111122. doi: 10.1016/j.jom.2014.11.002

Aqlan, F., \& Lam, S. S. (2015). A fuzzy-based integrated framework for supply chain risk assessment. International Journal of Production Economics, 161, 54-63. doi: 10.1016/j. ijpe.2014.11.013

Barbosa, M. W., \& Vicente, A. de la C. (2018). Managing supply chain resources with Big Data Analytics: A systematic review. International Journal of Logistics Research and Applications, 21(3), 177-200. doi: 10.1080/13675567.2017.1369501

Barratt, M. (2004). Understanding the meaning of collaboration in the supply chain. Supply Chain Management: An International Journal, 9(1), 30-42. doi: 10.1108/13598540410517566

Barratt, M., \& Oke, A. (2007). Antecedents of supply chain visibility in retail supply chains: A resource-based theory perspective. Journal of Operations Management, 25(6), 12171233. doi: 10.1016/j.jom.2007.01.003

Brandon-Jones, E., Squire, B., Autry, C. W., \& Petersen, K. J. (2014). A contingent resource-based perspective of supply chain resilience and robustness. Journal of Supply Chain Management, 50(3), 55-73. doi: 10.1111/jscm.12050

Bronzo, M., Resende, P. T. V. de, Oliveira, M. P. V. de, McCormack, K. P., Sousa, P. R. de, \& Ferreira, R. L. (2013). Improving performance aligning business analytics with process orientation. International Journal of Information Management, 33(2), 300-307. doi: 10.1016/j.ijinfomgt.2012.11.011

Brusset, X., \& Teller, C. (2017). Supply chain capabilities, risks, and resilience. International Journal of Production Economics, 184, 59-68. doi: 10.1016/j.ijpe.2016.09.008

Cao, G., Duan, Y., \& Li, G. (2015). Linking business analytics to decision making effectiveness: A path model analysis. IEEE Transactions on Engineering Management, 62(3), 384-395. doi: 10.1109/TEM.2015.2441875

Cao, M., \& Zhang, Q. (2011). Supply chain collaboration: Impact on collaborative advantage and firm performance. Journal of Operations Management, 29(3), 163-180. doi: 10.1016/j. jom.2010.12.008

Caridi, M., Crippa, L., Perego, A., Sianesi, A., \& Tumino, A. (2010). Measuring visibility to improve supply chain performance: A quantitative approach. Benchmarking: An International Journal, 17(4), 593-615. doi: 10.1108/14635771011060602

Caridi, M., Moretto, A., Perego, A., \& Tumino, A. (2014). The benefits of supply chain visibility: A value assessment model. International Journal of Production Economics, 151, 1-19. doi: 10.1016/j.ijpe.2013.12.025

Caridi, M., Perego, A., \& Tumino, A. (2013). Measuring supply chain visibility in the apparel industry. Benchmarking: An International Journal, 20(1), 25-44. doi: 10.1108/14635771311299470

Ceryno, P. S., Scavarda, L. F., Klingebiel, K., \& Yüzgülec, G. (2013). Supply chain risk management: A content analysis approach. International Journal of Industrial Engineering and Management, 4(3), 141-150. Recuperado de http://www.iim. ftn.uns.ac.rs/images/journal/volume4/ijiem_vol4_no3_6. pdf

Chae, K., Olson, D., \& Sheu, C. (2014). The impact of supply chain analytics on operational performance: A resource-based view. International Journal of Production Research, 52(16), 46954710. doi: 10.1080/00207543.2013.861616

Christopher, M., \& Peck, H. (2004). Building the resilient supply chain. International Journal of Logistics Management, 15(2), 1-13. doi: 10.1108/09574090410700275

CNN (2019). Domino's stockpiling pizza ingredients ahead of "disorderly" Brexit. Recuperado de https://www.gnews.com. $\mathrm{au} /$ world/dominos-pizza-chains-stockpiling-ingredientsahead-brexit-crash-uk-news/48061c6b-obdc-4d58-83db50060923dad3

Colicchia, C., Dallari, F., \& Melacini, M. (2010). Increasing supply chain resilience in a global sourcing context. Production Planning \& Control, 21(7), 680-694. doi: 10.1080/09537280903551969 
Colquitt, J. A., \& Zapata-Phelan, C. P. (2007). Trends in theory building and theory testing: A five-decade study of the Academy of Management Journal. Academy of Management Journal, 50(6), 1281-1303. doi: 10.5465/amj.2007.28165855

Continuity Central.com, (2018). Gartner highlights 'digital twins' as an emerging organizational resilience tool. . Recuperado de https://www.continuitycentral.com/index.php/news/ resilience-news/3560-gartner-highlights-digital-twins-as-anemerging-organizational-resilience-tool

Cosgrove, E. (2020). Smithfield closes more plants as coronavirus cases grow among workers. Recuperado de https://www. supplychaindive.com/news/coronavirus-smithfield-plantclose/575903/

Daugherty, P. J., Richey, R. G., Roath, A. S., Min, S., Chen, H., Arndt, A. D., \& Genchev, S. E. (2006). Is collaboration paying off for firms? Business Horizons, 49(1), 61-70. doi: 10.1016/j. bushor.2005.06.002

Davenport, T. H., Harris, J. G., Long, D. W. De, \& Jacobson, A. L. (2001). Data to knowledge to results: Building an analytics capability. California Management Review, 43(2), 117-138. doi: $10.2307 / 41166078$

Dubey, R., Gunasekaran, A., Childe, S. J., Fosso Wamba, S., Roubaud, D., \& Foropon, C. (2019). Empirical investigation of data analytics capability and organizational flexibility as complements to supply chain resilience. International Journal of Production Research, 59(1), 1-19. doi: 10.1080/00207543.2019.1582820

Durach, C. F., \& Machuca, J. A. D. (2018). A matter of perspective: The role of interpersonal relationships in supply chain risk management. International Journal of Operations \& Production Management, 38(10), 1866-1887. doi: 10.1108/ IJOPM-03-2017-0157

Graeml, A. R., Peinado, J. (2014). O efeito das capacidades logísticas na construção de resiliência da cadeia de suprimentos. Revista de Administração, 49(4), 642-655. doi: $10.5700 /$ rausp1174

Hair, J. F., Anderson, R. E., Tatham, R. L., Black, W. C., Babin, B. J., \& Anderson, R. E. (2009). Multivariate data analysis: Pearson Education Ltd. (7th ed.). Upper Saddle River, NJ: Prentice Hall. doi: 10.1016/j.ijpharm.2011.02.019

Hair, J. F., Jr., Hult, G. T. M., Ringle, C. M., \& Sarstedt, M. (2017). A primer on Partial Least Squares Structural Equation Modeling (PLS-SEM). In T. Oaks (Ed.), Handbook of market research (2nd ed., Vol. 26 1-374). Thousand Oaks, Los Angeles: Sage. doi: 10.1007/978-3-319-05542-8_15-1

Hallikas, J., Karvonen, I., Pulkkinen, U., Virolainen, V. M., \& Tuominen, M. (2004). Risk management processes in supplier networks. International Journal of Production Economics, 90(1), 47-58. doi: 10.1016/j.ijpe.2004.02.007

Hernandez, D. F., \& Haddud, A. (2018). Value creation via supply chain risk management in global fashion organizations outsourcing production to China. Journal of Global Operations and Strategic Sourcing, 11(2), 250-272. doi: 10.1108/ JGOSS-09-2017-0037
Holcomb, M. C., Ponomarov, S. Y., \& Manrodt, K. B. (2011). The relationship of supply chain visibility to firm performance. Supply Chain Forum: An International Journal, 12(2), 32-45. doi: $10.1080 / 16258312.2011 .11517258$

Ittmann, H. W. (2015). The impact of big data and business analytics on supply chain management. Journal of Transport and Supply Chain Management, 9(1), 1-9. doi: 10.4102/jtscm.vgi1.165

Ivanov, D., Dolgui, A., Das, A., \& Sokolov, B. (2019). Digital supply chain twins: Managing the ripple effect, resilience, and disruption risks by data-driven optimization, simulation, and visibility. In $1 \mathrm{ed}$. Handbook of ripple effects in the supply chain (pp. 309-332). Gewerbestrasse, Springer. https://doi. org/10.1007/978-3-030-14302-2_15

Jüttner, U., \& Maklan, S. (2011). Supply chain resilience in the global financial crisis: An empirical study. Supply Chain Management: An International Journal, 16(4), 246-259. doi: 10.1108/13598541111139062

Jüttner, U., Peck, H., \& Christopher, M. (2003). Supply chain risk management: Outlining an agenda for future research. International Journal of Logistics: Research and Applications, 6(4), 197-210. doi: 10.1080/13675560310001627016

Kirchoff, J.. (2019). How to assess risks in a globalized supply chain. Recuperado de https://www.supplychaindive.com/ news/assess-risks-globalized-supply-chain/568971/

Kohli, A. S., \& Jensen, J. B. (2010). Assessing effectiveness of supply chain collaboration: An empirical study. Supply Chain Forum: An International Journal, 11(2), 2-16. doi: $10.1080 / 16258312.2010 .11517228$

Kumar, S., \& Anbanandam, R. (2019). Impact of risk management culture on supply chain resilience: An empirical study from Indian manufacturing industry. Proceedings of the Institution of Mechanical Engineers, Part O: Journal of Risk and Reliability, 234(2), 246-259. doi: 10.1177/1748006X19886718

Kwak, D.-W., Seo, Y.-J., \& Mason, R. (2018). Investigating the relationship between supply chain innovation, risk management capabilities and competitive advantage in global supply chains. International Journal of Operations \& Production Management, 38(1), 2-21. doi: 10.1108/ IJOPM-06-2015-0390

Lacerda, R. T. de O., Ensslin, L., \& Ensslin, S. R. (2012). Uma análise bibliométrica da literatura sobre estratégia e avaliação de desempenho. Gestão \& Produção, 19(1), 59-78. doi: $10.1590 /$ s0104-530x2012000100005

Ladeira, M. B., Resende, P. T. V. de, Oliveira, M. P. V. de, McCormack, K., Sousa, P. R. de, \& Ferreira, R. L. (2016). Os efeitos da abordagem analítica e da gestão orientada para processos sobre o desempenho organizacional de micro e pequenas empresas brasileiras dos setores da indústria e de serviços. Gestão \& Produção, 23(32), 486-502. doi: 10.1590/ S0104-530X2012000200012

Laursen, G. H. N., \& Thorlund, J. (2010). Business analytics for managers. Hoboken, NJ: John Wiley \& Sons, Inc. https://doi. org/10.1002/9781118983812 
Li, G., Fan, H., Lee, P. K. C., \& Cheng, T. C. E. (2015). Joint supply chain risk management: An agency and collaboration perspective. International Journal of Production Economics, 164, 83-94. doi: 10.1016/j.ijpe.2015.02.021

Lopez, E. (2018). H\&M's turnaround runs through its supply chain. Recuperado de https://www.supplychaindive.com/ news/HM-turnaround-runs-through-supply-chain/520495/

Mandal, S., Sarathy, R., Korasiga, V. R., Bhattacharya, S., \& Dastidar, S. G. (2016). Achieving supply chain resilience: The contribution of logistics and supply chain capabilities. International Journal of Disaster Resilience in the Built Environment, 7(5), 544-562. doi: 10.1108/IJDRBE-04-2016-0010

Marafon, A. D., Ensslin, L., Lacerda, R. T. de O., \& Ensslin, S. R. (2015). The effectiveness of multi-criteria decision aid methodology: A case study of R\&D management. European Journal of Innovation Management, 18(1), 89-109. doi: 10.1108/EJIM-10-2013-0106

Merschmann, U., \& Thonemann, U. W. (2011). Supply chain flexibility, uncertainty and firm performance: An empirical analysis of German manufacturing firms. International Journal of Production Economics, 130(1), 43-53. doi: 10.1016/j. ijpe.2010.10.013

Namdar, J., Li, X., Sawhney, R., \& Pradhan, N. (2018). Supply chain resilience for single and multiple sourcing in the presence of disruption risks. International Journal of Production Research, 56(6), 2339-2360. doi: 10.1080/00207543.2017.1370149

Nooraie, S. V., \& Parast, M. M. (2015). A multi-objective approach to supply chain risk management: Integrating visibility with supply and demand risk. International Journal of Production Economics, 161, 192-200. doi: 10.1016/j.ijpe.2014.12.024

Norrman, A., \& Jansson, U. (2004). Ericsson's proactive supply chain risk management approach after a serious sub-supplier accident. International Journal of Physical Distribution \& Logistics Management, 34, 434-456. doi: 10.1108/09600030410545463

Ojha, R., Ghadge, A., Tiwari, M. K., \& Bititci, U. S. (2018). Bayesian network modelling for supply chain risk propagation. International Journal of Production Research, 56(17), 5795 5819. doi: 10.1080/00207543.2018.1467059

Oliveira, M. P. V. de, \& Handfield, R. (2019). Analytical foundations for development of real-time supply chain capabilities. International Journal of Production Research, 57(5), 1571-1589. doi: 10.1080/00207543.2018.1493240

Papadopoulos, T., Gunasekaran, A., Dubey, R., Altay, N., Childe, S. J., \& Fosso-Wamba, S. (2017). The role of Big Data in explaining disaster resilience in supply chains for sustainability. Journal of Cleaner Production, 142, 1108-1118. doi: 10.1016/j.jclepro.2016.03.059

Pavlov, A., Ivanov, D., Dolgui, A., \& Sokolov, B. (2018). Hybrid fuzzy-probabilistic approach to supply chain resilience assessment. IEEE Transactions on Engineering Management, 65(2), 303-315. doi: 10.1109/TEM.2017.2773574

Pettit, T. J., Croxton, K. L., \& Fiksel, J. (2019). The evolution of resilience in supply chain management: $A$ retrospective on ensuring supply chain resilience. Journal of Business Logistics, 40(1), 56-65. doi: 10.1111/jbl.12202
Pettit, T. J., Fiksel, J., \& Croxton, K. L. (2010). Ensuring supply chain resilience: Development of a conceptual framework. Journal of Business Logistics, 31(1), 1-21. doi: 10.1002/j.21581592.2010.tboo125.x

Ponomarov, S. Y., \& Holcomb, M. C. (2009). Understanding the concept of supply chain resilience. The International Journal of Logistics Management, 20(1), 124-143. doi: 10.1108/09574090910954873

Prakash, S., Gautam, A., \& Soni, U. (2018). Supply chain risk management and quality: A case study and analysis of Indian automotive industry. International Journal of Intelligent Enterprise, 5(1/2), 194. doi: 10.1504/IJIE.2018.10012158

Rajesh, R. (2017). Technological capabilities and supply chain resilience of firms: A relational analysis using Total Interpretive Structural Modeling (TISM). Technological Forecasting and Social Change, 118, 161-169. doi: 10.1016/j. techfore.2017.02.017

Ribeiro, J. P., \& Barbosa-Povoa, A. (2018). Supply chain resilience: Definitions and quantitative modelling approaches - A literature review. Computers and Industrial Engineering, 115, 109-122. doi: 10.1016/j.cie.2017.11.006

Ringle, C. M., Wende, S., \& Becker, J.-M. (2014). SmartPLS 3.0. Hamburg, Germany: SmartPLS.

Roberson, C. M. (2019). Preparing for the unknown in your supply chain. Recuperado de https://www.forbes.com/ sites/cathymorrowroberson/2019/10/07/preparing-for-theunknown-in-your-supply-chain/\#385eod6339ff

Sáenz, M. J., Revilla, E., \& Acero, B. (2018). Aligning supply chain design for boosting resilience. Business Horizons, 61(3), 443452 doi: 10.1016/j.bushor.2018.01.009

Sánchez, A., M. \& Pérez, M. P. (2005). Supply chain flexibility and firm performance. International Journal of Operations \& Production Management, 25(7), 681-700. doi: 10.1108/01443570510605090

Scavarda, L. F., Ceryno, P. S., Pires, S., \& Klingebiel, K. (2015). Supply chain resilience analysis: A Brazilian automotive case. RAE-Revista de Administração de Empresas, 55(3), 304-313. doi: $10.1590 /$ So034-759020150306

Scholten, K., Schilder, S.. (2015). The role of collaboration in supply chain resilience. Supply Chain Management: An International Journal, 20(4), 471-484. doi: 10.1108/SCM-112014-0386

Scholten, K., Scott, P. S., \& Fynes, B. (2014). Mitigation processes: Antecedents for building supply chain resilience. Supply Chain Management: An International Journal, 19(2), 211-228. doi: 10.1108/SCM-06-2013-0191

SEBRAE (2016). Anuário do trabalho nos pequenos negócios. Recuperado de https://m.sebrae.com.br/Sebrae/Portal Sebrae/Anexos/Anuario do Trabalho nos Pequenos Negócios 2016_pdf

Sheffi, Y., \& Rice, J. B., Jr. (2005). A supply chain view of the resilient enterprise. MIT Sloan Management Review, 47(1), 41-48. Recuperado de https://sloanreview.mit.edu/article/asupply-chain-view-of-the-resilient-enterprise 
Singh, N. P., \& Singh, S. (2019). Building supply chain risk resilience: Role of big data analytics in supply chain disruption mitigation. Benchmarking, 26(7), 2318-2342. doi: 10.1108/ BIJ-10-2018-0346

Sodhi, M. S., Son, B.-G., \& Tang, C. S. (2012). Researchers' perspectives on supply chain risk management. Production and Operations Management, 21(1), 1-13. doi:10.1111/j.19375956.2011.01251.x

Souza, G. C. (2014). Supply chain analytics. Business Horizons, 57(5), 595-605. doi: 10.1016/j.bushor.2014.06.004

Srinivasan, R., \& Swink, M. (2018). An investigation of visibility and flexibility as complements to supply chain analytics: An organizational information processing theory perspective. Production and Operations Management, 27(10), 1849-1867. doi: $10.1111 /$ poms.12746

Swafford, P. M., Ghosh, S., \& Murthy, N. (2006). The antecedents of supply chain agility of a firm: Scale development and model testing. Journal of Operations Management, 24(2), 170-188. doi: $10.1016 /$ j.jom.2005.05.002

Swafford, P. M., Ghosh, S., \& Murthy, N. (2008). Achieving supply chain agility through IT integration and flexibility. International Journal of Production Economics, 116(2), 288 297. doi: 10.1016/j.ijpe.2008.09.002

Tang, O., \& Musa, S. N. (2011). Identifying risk issues and research advancements in supply chain risk management. International Journal of Production Economics, 133(1), 25-34. doi: 10.1016/j.ijpe.2010.06.013

Teo, T. S. H., Nishant, R., \& Koh, P. B. L. (2016). Do shareholders favor business analytics announcements? Journal of Strategic Information Systems, 25(4), 259-276. doi: 10.1016/j. jsis.2016.05.001

Thomé, A. M. T., Scavarda, L. F., Pires, S. R. I., Ceryno, P., \& Klingebiel, K. (2014). A multi-tier study on supply chain flexibility in the automotive industry. International Journal of Production Economics, 158, 91-105. doi: 10.1016/j. ijpe.2014.07.024

Trkman, P., Mccormack, K., Oliveira, M. P. V. de, \& Ladeira, M. B. (2010). The impact of business analytics on supply chain performance. Decision Support Systems, 49(3), 318-327. doi: $10.1016 / j . d s s .2010 .03 .007$
Tummala, R., \& Schoenherr, T. (2011). Assessing and managing risks using the Supply Chain Risk Management Process (SCRMP). Supply Chain Management: An International Journal, 16(6), 474-483. doi: 10.1108/13598541111171165

Urciuoli, L., \& Hintsa, J. (2018). Improving supply chain risk management: Can additional data help? International Journal of Logistics Systems and Management, 30(2), 195. doi: 10.1504/IJLSM.2018.091962

Vanpoucke, E., Vereecke, A., \& Wetzels, M. (2014). Developing supplier integration capabilities for sustainable competitive advantage: A dynamic capabilities approach. Journal of Operations Management, 32(7-8), 446-461. doi:10.1016/j. jom.2014.09.004

Wieland, A., \& Wallenburg, C. M. (2013). The influence of relational competencies on supply chain resilience: a relational view. International Journal of Physical Distribution \& Logistics Management, 43(4), 300-320. doi: 10.1108/ ijpdlm-08-2012-0243

Wollenhaupt, G. (2019). In disaster response, health supply chain egos melt away. Recuperado de https://www. supplychaindive.com/news/pharma-healthcare-disasterresponse-hurricane/555942/

Wong, C. W. Y., Lirn, T. C., Yang, C. C., \& Shang, K. C. (2019). Supply chain and external conditions under which supply chain resilience pays: An organizational information processing theorization. International Journal of Production Economics, 226, 107610 doi: 10.1016/j.ijpe.2019.107610

Xu, S., Zhang, X., Feng, L., \& Yang, W. (2020). Disruption risks in supply chain management: A literature review based on bibliometric analysis. International Journal of Production Research, 59(11), 1-19. doi: 10.1080/00207543.2020.1717011

Zhu, S., Song, J., Hazen, B. T., Lee, K., \& Cegielski, C. (2016). How supply chain analytics enables operational supply chain transparency. International Journal of Physical Distribution \& Logistics Management, 48(1), 47-68. doi: 10.1108/ IJPDLM-11-2017-0341

Zineb, E., Brahim, B., \& Houdaifa, A. (2017). The impact of SCRM strategies on supply chain resilience: A quantitative study in the Moroccan manufacturing industry. International Journal of Supply Chain Management, 6(4), 70-75. Recuperado de http://ijis-scm.bsne.ch/ojs.excelingtech.co.uk/index.php/ IJSCM/article/download/1665/1665-6369-1-PB.pdf

\section{CONTRIBUIÇÃO DE AUTORIA}

Murilo Zamboni Alvarenga, Marcos Paulo Valadares de Oliveira e Hélio Zanquetto Filho trabalharam na conceitualização e abordagem teórica-metodológica. A revisão teórica possui contribuição de todos os autores. A coleta de dados foi coordenada por Marcos Paulo Valadares de Oliveira e Hélio Zanquetto Filho, e realizada por Murilo Zamboni Alvarenga. A análise dos dados foi realizada por Murilo Zamboni Alvarenga e auxiliada por Marcos Paulo Valadares de Oliveira e Hélio Zanquetto Filho. Murilo Zamboni Alvarenga, Marcos Paulo Valadares de Oliveira, Hélio Zanquetto Filho, Kevin C. Desouza e Paula Santos Ceryno participaram da redação e revisão final do manuscrito. 